\title{
SUSCEPTIBILITY OF ADULT HELIGMOSOMOIDES POLYGYRUS TO INTESTINAL INFLAMMATORY RESPONSES INDUCED BY HETEROLOGOUS INFECTION
}

\author{
Jerzy M. Behnke, ${ }^{*} \dagger$ Wladyslaw Cabaj $\ddagger$ and Derek Wakelin* \\ *MRC Experimental Parasitology Research Group, Department of Life Science, University of Nottingham, University Park, \\ Nottingham NG7 2RD, U.K. \\ $\ddagger W$. Stefanski Institute of Parasitology of the Polish Academy of Sciences, Pasteura 3, P.O. Box 153, 00-973 Warszawa, \\ Poland
}

(Received 14 January 1991; accepted 31 July 1991)

\begin{abstract}
Behnke J. M., Cabaj W. and Wakelin D. 1992. Susceptibility of adult Heligmosomoides polygyrus to intestinal inflammatory responses induced by heterologous infection. International Journal for Parasitology 22: 75-86. Adult $H$. polygyrus are capable of surviving for many months after primary exposure of mice to infective larvae, raising the possibility that worms of this species have inherent resistance to intestinal immune responses. Accordingly experiments were carried out to determine whether $H$. polygyrus are resistant to the inflammatory changes elicited during the acute phase of the intestinal response to Trichinella spiralis. Adult worms were expelled from mice when their presence coincided with the most intense phase of inflammation elicited by $T$. spiralis. The effect was dose-dependent with more intense $T$. spiralis challenge resulting in a correspondingly greater loss of $H$. polygyrus. Even the less pathogenic species $T$. pseudospiralis elicited a response of sufficient intensity in NIH mice to cause the expulsion of $H$. polygyrus from concurrently infected animals. Tissue larval stages of $H$. polygyrus were protected from expulsion by their location deep in the intestinal walls and the maximum detrimental effect against $H$. polygyrus was observed during the adult phase or during the establishment of $\mathrm{L} 3$ larvae. Acceleration of the response to $T$. spiralis in immune challenged mice resulted in earlier loss of $H$. polygyrus. When the expulsion of $T$. spiralis was delayed (e.g. from slow responder C57BL/10 mice) the loss of $H$.polygyrus took place correspondingly later. These experiments demonstrate unequivocally that mouse strains which normally tolerate chronic infections with $H$. polygyrus have the capacity to mount intestinal inflammatory responses of sufficient vigour to remove the worms but that this potential is not normally realized. However, the observation that some $H$. polygyrus always survived even when the response induced by $T$. spiralis was of the rapid secondary type suggests that the parasites are resilient in the face of the inflammatory response capable of removing most of the worms. It is suggested that in addition to the immunomodulatory strategy employed by adult worms to prevent the intestinal response being elicited, the worms have a second line of defence which is reflected in their resilience to responses which they have been unable to prevent.
\end{abstract}

INDEX KEY WORDS: Heligmosomoides polygyrus; Nematospiroides dubius; Trichinella spiralis; Trichinella pseudospiralis; Nematoda; mouse; intestinal inflammation; immunity; evasion of immunity.

\section{INTRODUCTION}

THE intestinal inflammatory response elicited by Nippostrongylus brasiliensis and Trichinella spiralis has been the focus of attention for well over 4 decades and the immunological control of this response is well documented (Rothwell, 1989; Moqbel \& MacDonald, 1990). It is established that the induction of the response is specific, requiring the sensitization of $\mathrm{CD}^{+} \mathrm{T}_{\mathrm{H}}$ lymphocytes (Crook, 1990; Grencis, Riedlinger \& Wakelin, 1985), however, the final effectors remain as controversial as ever. Among recent additions to the list of possible effectors are leukotrienes (Douch,

$\uparrow$ To whom all correspondence should be addressed.
Harrison, Buchanan \& Greer, 1983), platelet activating factor (Moqbel \& MacDonald, 1990), tumour necrosis factor (Ovington, 1987), mast cell protease II (McKean \& Pritchard, 1989) and oxygen radicals (Smith \& Bryant, 1989a,b). Once induced the specifically triggered effectors act non-specifically with detrimental consequences to unrelated parasites residing in the intestine at the time of expression (Christensen, Nansen, Fagbemi \& Monrad, 1987). During the response to $T$. spiralis, totally unrelated nematodes (Nippostrongylus brasiliensis, see Kennedy, 1980) and cestodes ( $\mathrm{Hy}$ menolepis diminuta, see Behnke, Bland \& Wakelin, 1977; Christie, Wakelin \& Wilson, 1979; H. nana, see Ferretti, Gabriele, Palmas \& Wakelin, 1984) may be rejected and others ( $H$. microstoma, see Howard, 
Christie, Wakelin, Wilson \& Behnke, 1978), may have their growth severely impaired. None of these show specific immunological cross immunity with $T$. spiralis and their loss from the host is brought about solely through the non-specific effectors of the inflammatory response initiated by $T$. spiralis and the resultant changes in the intestinal environment.

Not all nematodes parasitizing the intestines of vertebrates elicit acute inflammatory responses of the sort associated with the rejection of $T$. spiralis and $N$. brasiliensis. Some cause long-term chronic infections in which there is little/no evidence of hostprotective immunity. For example, Trichostrongylus tenuis accumulates in wild grouse and survives for over 600 days (Shaw \& Moss, 1989), Necator americanus lives for up to 17 years in man (Palmer, 1955; Beaver, 1988) and Heligmosomoides polygyrus (Nematospiroides dubius) causes infections which last for 8-10 months in mice (Robinson, Wahid, Behnke \& Gilbert, 1989). Little is known about the survival strategies of the former species, but $H$. polygyrus has been intensively investigated and it is generally accepted that this species is immunomodulatory, downregulating intestinal immunity to enable its own survival (Behnke, 1987). One of the indicators of intestinal inflammation, the mast cell response, is hardly detectable in mice carrying $H$. polygyrus and even in mice concurrently infected with $T$. spiralis mastocytosis is greatly depressed (Dehlawi, Wakelin \& Behnke, 1987). This in turn is associated with prolonged infection with $T$. spiralis (Behnke, Wakelin \& Wilson, 1978) indicating that $H$. polygyrus not only downregulates the inflammatory response in relation to its own survival but that the mechanism it invokes acts non-specifically and that it can benefit unrelated species resident in concurrently infected animals (Behnke, 1987; Christensen et al., 1987).

The effect of $H$. polygyrus in prolonging concurrent infections with heterologous species of parasites is well documented (Behnke, 1987; Christensen et al., 1987; Cabaj, 1989). However, the effect of an intense inflammatory response on $H$. polygyrus has never been studied in detail, although the worms are known to be resistant to free oxygen radical damage in vitro (Smith \& Bryant, 1986). Furthermore, in comparison with nematodes causing acute infections, $H$. polygyrus have relatively high levels of oxygen radical scavenging enzymes and it has bcen suggested that in consequence the worms are more resilient to host-generated free oxygen radical-mediated damage, which may be a component of the non-specifically acting effectors of intestinal expulsion (Smith \& Bryant, 1986). Thus in addition to showing immunomodulatory properties $H$. polygyrus may have a second line of defence to safeguard their survival in the host.
In the present paper we report the results of experiments which extend our earlier studies but this time examine the effect of the intestinal inflammatory response elicited by $T$. spiralis on concurrent infection with $H$. polygyrus. T.pseudospiralis, which is reputedly less pathogenic and causes longer lasting infections in mice than $T$. spiralis but also elicits an acute intestinal response (Przyjalkowski, Starzynski, Pykalo \& Cabaj, 1981; Przyjalkowski \& Pykalo, 1988; Stewart, Wood \& Boley, 1985; Stewart, Mann, Ubelaker, McCarthy \& Wood, 1988), was exploited as a further source of intestinal inflammation. The experiments were designed to minimize the possible immunodepressive influence of $H$. polygyrus, by keeping infections with the latter species low, worm burdens being just sufficient to allow any reductions to be recognized and analysed statistically, but not high enough to cause a major downregulation of the intestinal response to Trichinella spp. In contrast Trichinella infections were kept high to ensure maximum establishment and the induction of the host protective intestinal response. It is shown conclusively that adult $H$. polygyrus are lost when the parasites are caught in an inflammatory response which they have been unable to prevent. However, the same experiments again emphasize the tremendous resilience of this parasite, since expulsion of $H$. polygyrus was never complete and often only minimal, supporting the existence of a second line of defence against host immunity, as suggested by Smith \& Bryant (1986).

\section{MATERIALS AND METHODS}

Mice. The mice used in this study were purchased from Harlan Olac Ltd and were bred in the departmental animal house. Unless otherwise stated, male mice were used throughout. All animals were housed under conventional animal house conditions with access to food and water ad libitum.

Parasite. T. spiralis was originally obtained in 1975 from the Wellcome Research Laboratories (Beckenham, Kent) since when it has been passaged regularly in CFLP mice. Our stain corresponds to $H$. polygyrus bakeri as reported by Durette-Desset, Kinsella \& Forester (1972). The methods used for maintenance and infection have been reported previously (Jenkins \& Behnke, 1977). Adult parasites were recovered by a $6 \mathrm{~h}$ incubation at $37^{\circ} \mathrm{C}$ of small intestines, suspended in a gauze, in $50 \mathrm{ml}$ heakers containing Hanks's saline as described. Faecal egg counts were carried out by the method of Behnke \& Parish (1979). The strain of T. spiralis and the methods used for the infection of mice and recovery of worms have been described previously (Wakelin \& Lloyd, 1976). T. pseudospiralis was obtained from the Polish Academy of Sciences and was maintained identically to $T$. spiralis.

Statistical analysis of results. The results are presented as group mean values $(M W R) \pm$ standard error (S.E.M.). Nonparametric statistical procedures were used to analyse the data sets, because of small sizes (Sokal \& Rohlf, 1969). When more than two groups required comparison at a single time 

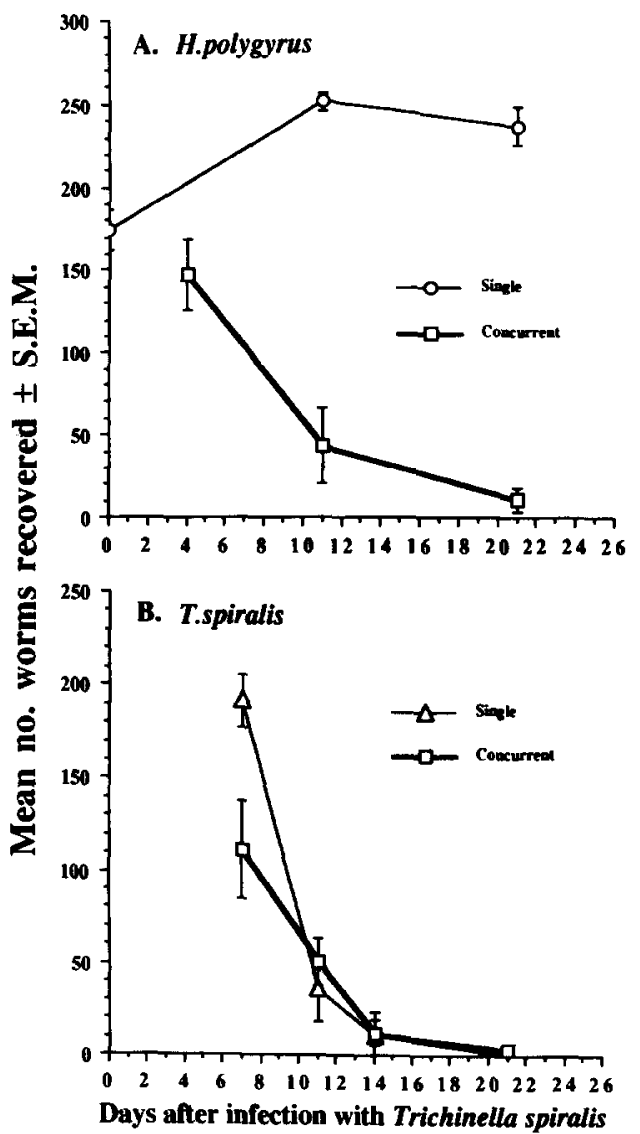

FIG. 1. Loss of adult $H$. polygyrus during the intestinal inflammatory phase of the response to concurrent $T$. spiralis infection (Expt 1). Three groups of male NIH mice were infected with $250 \mathrm{~L} 3$ of $H$. polygyrus (single infection) 14 days earlier (day -14), 330 muscle larvae of $T$. spiralis (single infection) on day 0 or both species (concurrent infection) and were killed for worm recovery on the days shown $(n=5$ on each occasion).

point the Kruskal-Wallis statistic $H$ was calculated to determine whether there was a significant treatment effect. If significant, specific groups were compared to the control group (or as stated) by the Mann-Whitney $U$ test. Correlations between variables were tested by the Spearman Rank Order Correlation test and the statistic $r_{s}$ is given, as appropriate. Probabilities were calculated from statistics tables and are presented as follows: ${ }^{*} P=0.05 ;{ }^{* *} 0.05>P$ $\geqslant 0.02 ;{ }^{* * *} 0.02>P \geqslant 0.01 ; * * * * 0.01>P \geqslant 0.001$; $* * * * * P<0.001$.

\section{RESULTS}

Expulsion of adult $\mathrm{H}$. polygyrus following superimposed challenge with Trichinella spiralis (Expt 1)

A preliminary experiment was carried out in which NIH mice were infected with $H$. polygyrus, $T$. spiralis or with both species so that $T$. spiralis was super- imposed on an existing adult population of $H$. polygyrus in the concurrently infected groups. It can be seen from the data which are summarized in Fig. 1 that the majority of $T$. spiralis were expelled at exactly the same time by mice carrying single and concurrent infections, although worms persisted longer in concurrently infected mice with $2.8 \pm 3.8$ being recovered on day 21 compared with none in the single infection group ( $T$. spiralis only). It is also evident from Fig. 1 that whilst $H$. polygyrus persisted without loss to day 21 post-challenge in mice infected only with this species, there was a considerable loss of worms from concurrently infected animals with an 82.9 and $95.6 \%$ reduction by days 11 and 21 , respectively.

Relationship between intensity of T. spiralis (Expts 2-5) and $\mathrm{T}$. pseudospiralis (Expts 6-7) infections and the proportion of $\mathrm{H}$. polygyrus lost during intestinal inflammation

Four separate experiments were carried out to ascertain the importance of infection intensity with $T$. spiralis on the proportion of adult $H$. polygyrus lost during the ensuing inflammatory response in concurrently infected mice. All the experiments were carried out in NIH mice, each included control groups to monitor the establishment of $T$. spiralis in single and concurrent infection groups. In each, $H$. polygyrus was given 14 days before $T$. spiralis and the mice were killed 3 weeks after the latter infection. The results are summarized in Fig. 2A, in which the mean worm burdens recovered at autopsy are expressed as a percentage of the single infection control group ( $H$. polygyrus only). Rank order correlation cocfficients were also calculated and these are presented in Table 1.

TABle 1-STATISTICAL ANALYSIS OF THE RelationshiP BETWEEN THE INTENSITY OF $T$. spiralis AND $T$. pseudospiralis INFECTION AND THE PROPORTION OF $\boldsymbol{H}$. polygyrus SURVIVING THE INTESTINAL INFLAMMATORY RESPONSE IN CONCURRENTLY INFECTED MICE

\begin{tabular}{lccc}
\hline Experiment & Number of mice & $r_{s}$ & $P$ \\
\hline Effect of $T$. spiralis & & & \\
2 & 40 & -0.792 & $<0.001$ \\
3 & 17 & -0.777 & $<0.001$ \\
4 & 30 & -0.919 & $<0.001$ \\
5 & 30 & -0.718 & $<0.001$ \\
Effect of $T$. pseudospiralis & & \\
6 & 25 & -0.815 & $<0.001$ \\
7 & 41 & -0.872 & $<0.001$ \\
& & &
\end{tabular}

Spearman Rank-Order Correlation Coefficients were calculated using standard procedures and probabilities were read from tables. 


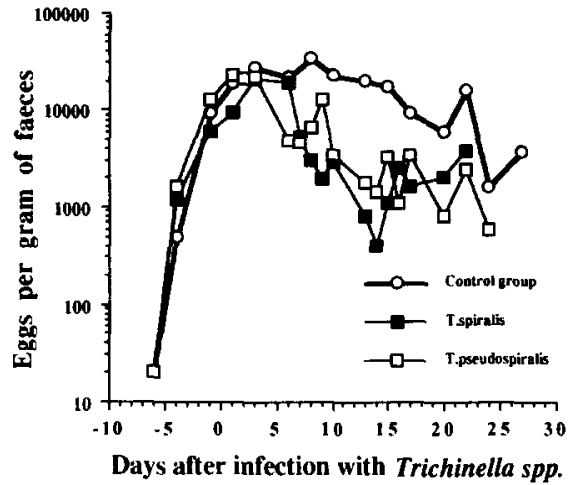

FIG. 3. The effect of the inflammatory response generated by Trichinella on the fecundity of $H$. polygyrus in concurrently infected mice (Expts 5 and 7). These experiments were carried out simultaneously and were based on the same batch of mice and the same infective larvae of $H$, polygyrus. The establishment of $T$. spiralis and $T$. pseudospiralis varied significantly following administration of identical doses of infective larvae. The figure therefore compares faecal egg counts in groups of concurrently infected mice $(n=6)$ in which establishment of Trichinella spiralis was $667.0 \pm 20$ and $T$. pseudospiralis $465 \pm 10$. The control group $(n=6)$ was the same for both experiments.

declined. The reduction in eggs per gram of faeces corresponded to the onset of the inflammatory response to Trichinella.

Consequence of varying the interval between and order of infection with $\mathrm{H}$. polygyrus and $\mathrm{T}$. spiralis (Expts 8-11)

All of the experiments described so far were based on a primary infection with $H$. polygyrus followed 14 days later by challenge with $T$. spiralis or $T$. pseudospiralis. The inflammatory response generated by Trichinella spp. will therefore have coincided in all cases with the presence of adult $H$. polygyrus. It was necessary to extend these observations to encompass the effect of inflammation on the larval stages of $H$. polygyrus. Four experiments were carried out in which the sequence of primary/challenge infection and the interval between primary and challenge were varied. The results from three such experiments are summarized in Fig. 4.

The trend is very similar in all three experiments, with almost identical results for two (Expts 8 and 9) and a slightly earlier sequence of events but a similarly shaped graph for Expt 10. The most impressive effect was always when $H$. polygyrus was administered well before $T$. spiralis such that the inflammatory response coincided with the presence of adult worms. As the interval between primary infection with $H$. polygyrus and challenge with $T$. spiralis was reduced, so the proportion of $H$.polygyrus surviving increased. When

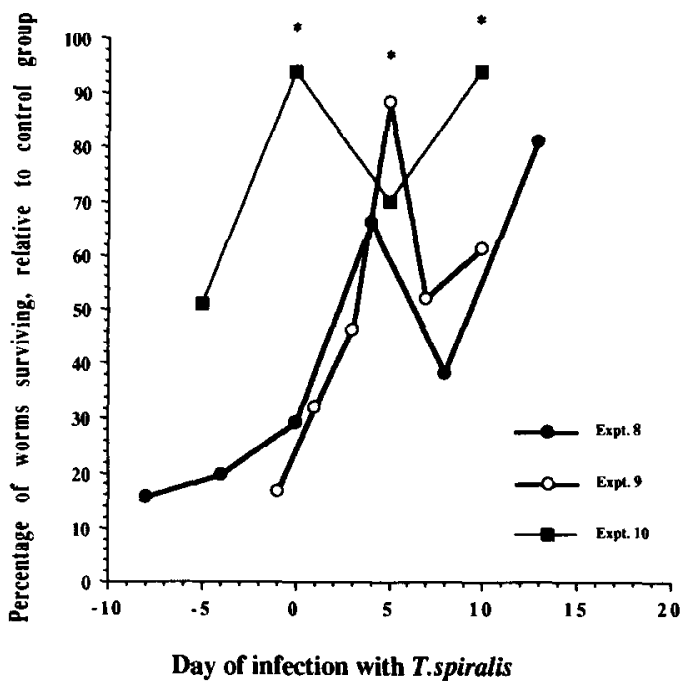

Fig. 4. The effect of varying the time interval between infections and the sequence of infections with $H$.polygyrus and $T$. spiralis on the survival of $H$. polygyrus in concurrently infected mice (Expts 8-10). Three experiments were carried out in malc NIH micc; 250 (Expts 9 and 10) or 400 musclc larvae (Expts 8) of $T$. spiralis were administered on day 0 . Groups of five to six mice were infected with $100 \mathrm{~L} 3$ of $H$. polygyrus before, on the same day or after $T$. spiralis on the days shown and on each occasion a group of naive control mice was also given $H$. polygyrus. All the mice were killed for worm counts on day 28 post- $T$. spiralis infection. The establishment of $T$. spiralis in single infection mice killed before expulsion (days, 3,6 and 6, respectively) was as follows: Expt 8, 247.3 \pm 19.6; Expt 9, $169 \pm 17$; Expt 10, $204.3 \pm 16.3$. The mean establishment of $H$. polygyrus in single infection control mice ranged from 73.2 to $80.7 \%$ (Expt 8), 80.5 to 91.8\% (Expt 9) and 77.6 to $100 \%$ (Expt 10) of the inoculum administered. Statistical analysis: $M W R$ s from concurrently infected groups were compared with their respective single infection control group by the MannWhitney $U$ test and points marked* were not significantly different from control groups $(P>0.05)$.

H. polygyrus was given 5 days after $T$. spiralis (Expts 8 and 9) or on the day of $T$. spiralis infection (Expt 10) relatively few worms were lost. However, as the interval between a primary $T$. spiralis infection and a challenge with $H$. polygyrus increased, so the proportion of worms surviving again decreased. Finally when the interval was 13 days (Expt 8), there was again relatively little loss of $H$. polygyrus.

A fourth experiment (Expt 11) examined the effect of administering the two species at the same time (day 0 ) and this confirmed the data from Expts 8 and 9, indicating that only $26 \%$ of the worms survived to day $21(M W R$ control group $=114.8 \pm 13.4, n=5 ; M W R$ concurrently infected group $=29.8 \pm 9.3, n=5$ ). 
TABLE 2-COMPARISON OF THE EFFECT OF PRIMARY AND SECONDARY RESPONSES TO Trichinella spiralis ON Heligmosomoides polygyrus (EXPERIMENT 12)

\begin{tabular}{|c|c|c|c|c|c|}
\hline \multicolumn{3}{|c|}{ Treatment } & \multirow[t]{2}{*}{$n$} & \multicolumn{2}{|c|}{ Mean no. worms recovered \pm S.E.M. on day $?$} \\
\hline $\begin{array}{l}\text { Primary }{ }^{\dagger} \\
T \text {. spiralis }\end{array}$ & H. polygyrus $\ddagger$ & $\begin{array}{c}\text { Secondary } \S \\
T . \text { spiralis }\end{array}$ & & H.polygyrus\| & T. spiralis \\
\hline No & Yes & No & 6 & $74.3 \pm 1.8$ & - \\
\hline Yes & Yes & No & 8 & $77.8 \pm 1.8$ & - \\
\hline No & Yes & Yes & 6 & $29.3 \pm 6.0^{* * * *}$ & $111.5 \pm 19.8$ \\
\hline Yes & Yes & Yes & 8 & $17.6 \pm 4.2^{* * * *}$ & $2.4 \pm 1.5$ \\
\hline No & No & Yes & 4 & - & $170.5 \pm 22.5$ \\
\hline Yes & No & Yes & 5 & - & $12.6 \pm 12.6$ \\
\hline
\end{tabular}

All the mice were male NIH.

$\dagger 440$ larvae of $T$. spiralis were given on day -34 .

$\ddagger 100$ larvae of $H$. polygyrus were administered on day -14 and all the mice were killed on day 7 , i.e. 7 days after challenge with $T$. spiralis.

$\$ 300$ larvae of $T$. spiralis were given on day 0 .

Statistical analysis of results:

$\|$ For $H$. polygyrus $H=21.706, P<0.001$.

If For $T$. spiralis $H=16.83, P<0.001$.

Additional comparisons were carried out with the Mann-Whitney $U$ test using the values for control mice infected only with $H$. polygyrus as the reference group.

$* * * * 0.01>P \geqslant 0.001$.

Comparison of the effect on $\mathrm{H}$. polygyrus of primary and secondary responses to $\mathrm{T}$. spiralis (Expts 12-14)

The secondary response to $T$. spiralis occurs earlier in immune NIH mice and is associated with more intense inflammation of the intestine than takes place during the primary response. If the principal reason for loss of $H$. polygyrus in concurrently infected mice is through the non-specifically acting mediators of the inflammatory response specifically elicited by $T$. spiralis, secondary responses to $T$. spiralis occurring in concurrently infected mice would be expected to exert a significantly more potent effect against $H$.polygyrus. This prediction was tested in three experiments. All were carried out in male NIH mice. The basic design involved four groups of mice to control for possible specific as well as non-specific interactions. Thus each experiment had one group of mice which received only $H$. polygyrus (day -14) and therefore controlled for infectivity of this species (infectivity control). A second group received a primary infection with $T$. spiralis (days -34 or -35 ) and a second with $H$. polygyrus (day -14) to control for possible cross immunity between the species as well as residual activity persisting after expulsion of the primary $T$. spiralis infection (cross immunity control). A third group received a primary infection with $H$. polygyrus (day -14 ) followed by challenge with $T$. spiralis (day $0)$ as in earlier experiments. The important experimental group comprised mice which received a primary infection with $T$. spiralis (day -34 or -35 ), followed by a second infection with $H$. polygyrus (day -14 ) and a third infection with $T$. spiralis (day 0 ). Additional control groups monitored the infectivity of $T$. spiralis in both infections and the expression of acquired resistance in the absence of concurrent infection.

The results from Expt 12 are summarized in Table 2. It is quite clear that there was no residual activity persisting from the primary $T$. spiralis and no cross immunity between the species, since the establishment of $H$. polygyrus was not impaired when $T$. spiralis was given 3 weeks earlier. However, both of the $H$. polygyrus infected groups challenged with $T$. spiralis on day 0 had significantly fewer $H$. polygyrus on day 7 and the $M W R$ was lower in mice which had been primed with $T$. spiralis. As can be seen, control groups indicated that the primary $T$. spiralis infection had sensitized the mice and an accelerated expulsion of $T$. spiralis ensued when both single-species and concurrently infected mice were subjected to homologous challenge.

A second experiment (Expt 13) also monitored changes in both species on two occasions following challenge with $T$. spiralis (days $5, H$. polygyrus, $H=4.718, P=$ not significant and $28, H$. polygyrus, $H=7.463, P=0.024)$. Control groups again established that the mice receiving the primary infection with $T$. spiralis on day -35 developed strong 
immunity to challenge administered on day $0 . H$. polygyrus established unimpaired in mice which had received $T$. spiralis on day -35 and were challenged with $H$. polygyrus 3 weeks later (day -14$)$. On day +5 (i.e. day 19 of the $H$. polygyrus infection) these mice had $84.3 \pm 4.3$ worms $(n=6)$ and on day +28 (42 days after infection with $H$. polygyrus) $80.3 \pm 6.6(n=6)$. The $T$. spiralis infection given on day 0 comprised 250 muscle larvae of which $144.5 \pm 4.1$ established and on this occasion, by day 5 , there was no effect on $H$. polygyrus in mice which had not been primed with $T$. spiralis ( $M W R=83.3 \pm 2.7, n=6)$ although a small reduction was evident by day $28(M W R=72.3 \pm 6.7$, not significant). However, mice primed with $T$. spiralis on day -35 , challenged with $H$.polygyrus on day -14 and then with $T$. spiralis on day 0 only had $57.5 \pm 9.8$ $H$. polygyrus on day 5 and $43.8 \pm 7.9$ on day $28(P=$ 0.008 ).

These two experiments were repeated on a larger scale with groups of mice from all the above treatments killed at regular intervals throughout the secondary infection with $T$. spiralis. The results are shown in Fig. 5. The data confirm and extend both of the above experiments and show that secondary responses to $T$. spiralis which take place in concurrently infected mice exert a rapid and potent effect against $H$. polygyrus causing earlier loss of worms than when a primary $T$. spiralis infection is superimposed on existing adult $H$. polygyrus.

Comparison of the effect on $\mathrm{H}$. polygyrus of primary responses to $\mathrm{T}$. spiralis in fast and slow responder mouse strains (Expts 15-17)

The final series of experiments exploited the known difference in the time of onset and subsequent intensity of the intestinal inflammatory response to $T$. spiralis between fast $(\mathrm{NIH})$ and slow $(\mathrm{C} 57 \mathrm{BL} / 10)$ responder strains (Wakelin, 1978), in a further attempt to link the loss of $H$. polygyrus from concurrently infected mice to the acute inflammatory response elicited by $T$. spiralis. It would be anticipated that concurrently infected NIH mice would lose a greater proportion of $H$. polygyrus and the loss would occur earlier than in C57BL/10 mice.

The predictions of the hypothesis were tested in three experiments, two of which are summarized in Table 3 (Expts 15 and 16). In Expt 15, a single infection dose of $T$. spiralis was used throughout, whereas in Expt 16, groups of concurrently infected mice were challenged with one of two doses as shown. There was also a difference in establishment as shown by control groups infected only with $T$. spiralis and killed before expulsion. Thus in Expt 15 establishment of $T$. spiralis was relatively poor, but despite this a significant reduction in the $H$. polygyrus worm burden was

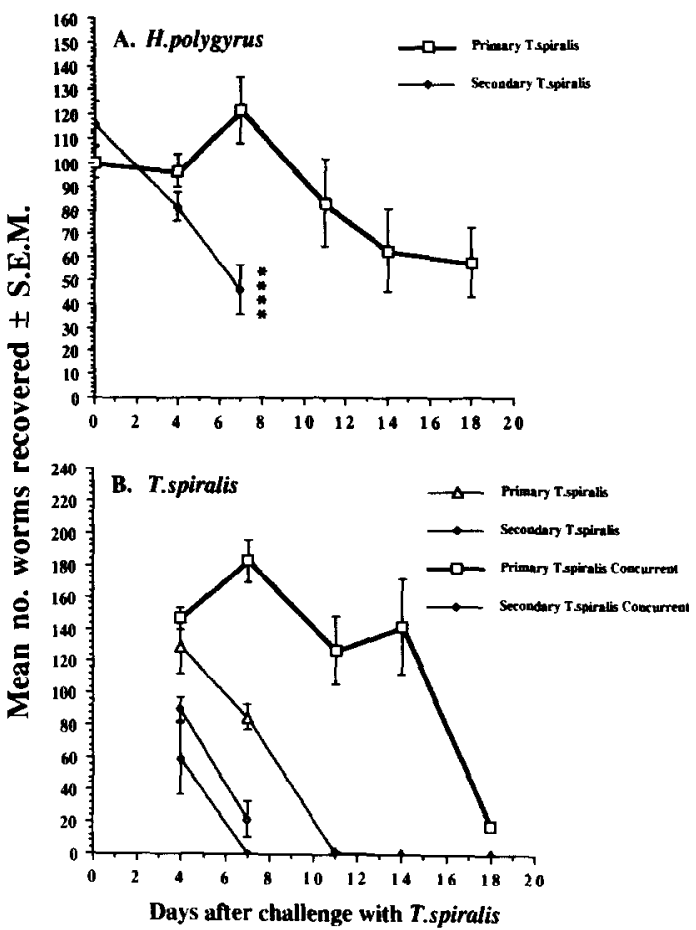

FIG. 5. Comparison of the effect on $H$. polygyrus of primary and secondary responses to $T$. spiralis (Expt 14). A. Groups of male NIH mice received a primary infection with 320 muscle larvae on day -35 , followed by $100 \mathrm{~L} 3$ of $H$. polygyrus on day -14 and 360 muscle larvae of $T$. spiralis on day 0 and were killed on the days shown $(n=9-10)$. One group of mice received only $H$. polygyrus and were killed on day 0 to monitor infectivity of the $H$. polygyrus inoculum (represented on the figure as primary infection day $0, n=9$ ). Another group received the primary $T$. spiralis infection (day -35), followed by $H$. polygyrus on day -14 but was not challenged with $T$. spiralis, to monitor cross immunity between the species (represented on the figure as secondary $T$. spiralis, day $0, n=10$ ). B. Additional control groups monitored the course of primary and secondary infections with $T$. spiralis in the presence ( $n=9-10$ on each occasion) and absence ( $n=4$ on each occasion) of $H$. polygyrus. Statistical analysis of results: The $M W R$ s were compared with control groups killed on the same day. For $T$. spiralis day $4, H=16.761, P=0.001$ and day $8, H=16.97, P=$ 0.001 . Additional comparisons were made using the Mann-

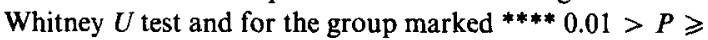
0.001 .

observed by day 28 post-challenge with $T$. spiralis in concurrently infected mice of both strains. However, proportionally the loss was marginally greater in NIH relative to $\mathrm{C} 57 \mathrm{BL} / 10$ mice $(33.9 \%$ vs $28.3 \%)$. When $T$. spiralis infections were more intense (Expt 16), significant loss of $H$. polvgyrus was detected as early as 11 days post-challenge confirming earlier data (Fig. 1). Proportionally the loss was again greater in NIH mice. 
TABle 3-Expllsion of Heligmosomoides polygyrus from FAST and SLOW RESPONDER STRAins CONCURRENTLy INFECTED WITH Trichinella spiralis

\begin{tabular}{|c|c|c|c|c|c|}
\hline \multirow[b]{3}{*}{ Strain } & \multirow{3}{*}{$\begin{array}{l}\text { Infection } \ddagger \\
\quad \text { with } \\
T \text {. spiralis }\end{array}$} & \multicolumn{4}{|c|}{ Mean no of $H$. polygyrus $\dagger$ recovered \pm S.E.M. on days after infection with $T$. spiralis $(n)$} \\
\hline & & & & & \\
\hline & & Day 0 & Day 28 & Day 11 & Day 30 \\
\hline NIH & None & $91.5 \pm 6.6(6)$ & $112.0 \pm 4.6(6)$ & $87.5 \pm 2.8(6)$ & $81.2 \pm 3.1(12)$ \\
\hline NIH & 400 larvae & - & $74.0 \pm 8 * 0(9)$ & $37.0 \pm 5.4(12)$ & $5.7^{* * * * *} \pm 2.9(6)$ \\
\hline NIH & 750 larvae & 一 & & $51 .{ }^{* * * *} \pm 8.7(11)$ & $16.8^{* * * * *} \pm 6.3(5)$ \\
\hline $\mathrm{C} 57 \mathrm{BL} / 10$ & None & $89.0 \pm 11.1(5)$ & $88.8 \pm 4.1(5)$ & $74.8 \pm 5.1(6)$ & $71.9 \pm 3.8(12)$ \\
\hline C57BL/10 & 400 larvae & - & $63.7 \pm 3.1(6)$ & $78.4 \pm 2.9(11)$ & $35.5 \stackrel{* * * *}{ \pm} 12.7(6)$ \\
\hline $\mathrm{C} 57 \mathrm{BL} / 10$ & 750 larvae & - & & $56.2 \stackrel{* * * *}{ \pm} 5.8(11)$ & $32.8 \pm 6.2(6)$ \\
\hline
\end{tabular}

$\uparrow$ All the mice were infected with $120 \mathrm{~L} 3$ of $H$. polygyrus on day -14 (Expt 15) or with $100 \mathrm{~L} 3$ on day -20 (Expt 16).

$\ddagger$ Mice infected with both species of parasites were given the dose of muscle larvae shown, on day 0 . Additional control groups were included to monitor the establishment of $T$. spiralis ( $n=3$ in all cases) and the following parasite burdens were recovered:

Expt 15 (day 5), NIH $=121.3 \pm 23.2$ and $\mathrm{C} 57 \mathrm{BL} / 10=111.3 \pm 14.8$.

Expt 16 (day 4), $400 T$. spiralis larvae, $\mathrm{NIH}=230.3 \pm 5.8$ and $\mathrm{C} 57 \mathrm{BL} / 10=247.3 \pm 42.8$.

750 T. spiralis larvae, $\mathrm{NIH}=457.7 \pm 6.4$ and $\mathrm{C} 57 \mathrm{BL} / 10=442.7 \pm 29.4$.

Statistical analysis of results:

Expt 16, day 11, for NIH mice $H=12.805(P=0.002)$; for C57BL $/ 10, H=9.881(P=0.007)$.

Day 30, for NIH mice $H=17.245(P<0.001)$; for C57BL $/ 10, H=10.707(P=0.005)$.

Additional comparisons were made using the Mann-Whitney $U$ test and the single species infection (H.polygyrus only) control group for reference.

${ }^{* * * *} 0.01>P \geqslant 0.001,{ }^{* * * * *} P<0.001$.

By day 30 the differences between the two strains were even further exaggerated with a 93 and $79.3 \%$ reduction in $H$. polygyrus in NIH mice compared with the singly infected control group, but a loss of only 50.6 and $54.4 \%$ in $\mathrm{C} 57 \mathrm{BL} / 10$ mice.

These findings were confirmed in a larger experiment which followed the time course of events more precisely in both mouse strains and the results are summarized in Figs. 6 and 7. Again it is quite evident that $H$. polygyrus burdens already began to decline by day 10 in the fast responder NIH mice, at a time when $T$. spiralis had not yet been lost (Fig. 6c). Then, as $T$. spiralis was expelled, so a major proportion of $H$. polygyrus was also lost. In C57BL/10 mice, inflammation generated by $T$. spiralis was slower to appear and correspondingly loss of both parasites in concurrently infected animals and $T$. spiralis in single infection control groups was delayed. As in the earlier experiments (Expts 15 and 16) the loss from NIH mice was proportionally greater (day $20, \mathrm{NIH}=79.5 \%$, $\mathrm{C} 57 \mathrm{BL} / 10=21.3 \%$ ). The difference in the onset and intensity of intestinal inflammation between the strains was also reflected in the effects it had on the fecundity of $H$. polygyrus in concurrently infected mice. Figure 7 shows that faecal egg counts declined in concurrently infected NIH mice as early as day 5 after challenge with $T$. spiralis whereas in $\mathrm{C} 57 \mathrm{BL} / 10$ mice no appreciable reduction was evident before day 14 postchallenge.

The effect of $\mathrm{H}$. polygyrus on the expulsion of $\mathrm{T}$. spiralis from concurrently infected mice

Although the primary purpose of this study was to examine the effect of the intestinal inflammation elicited by $T$. spiralis on the survival of $H$. polygyrus in concurrent infections, control groups were included in all experiments to monitor the infectivity of $T$. spiralis and to confirm that expulsion took place at the expected times. In concurrently infected mice, both $H$. polygyrus and $T$. spiralis were counted at autopsy, enabling a comparison of $T$. spiralis $M W R \mathrm{~s}$ between single and concurrent infection groups. In general the experiments were designed so as to minimize the influence of the known immunomodulatory effects of 

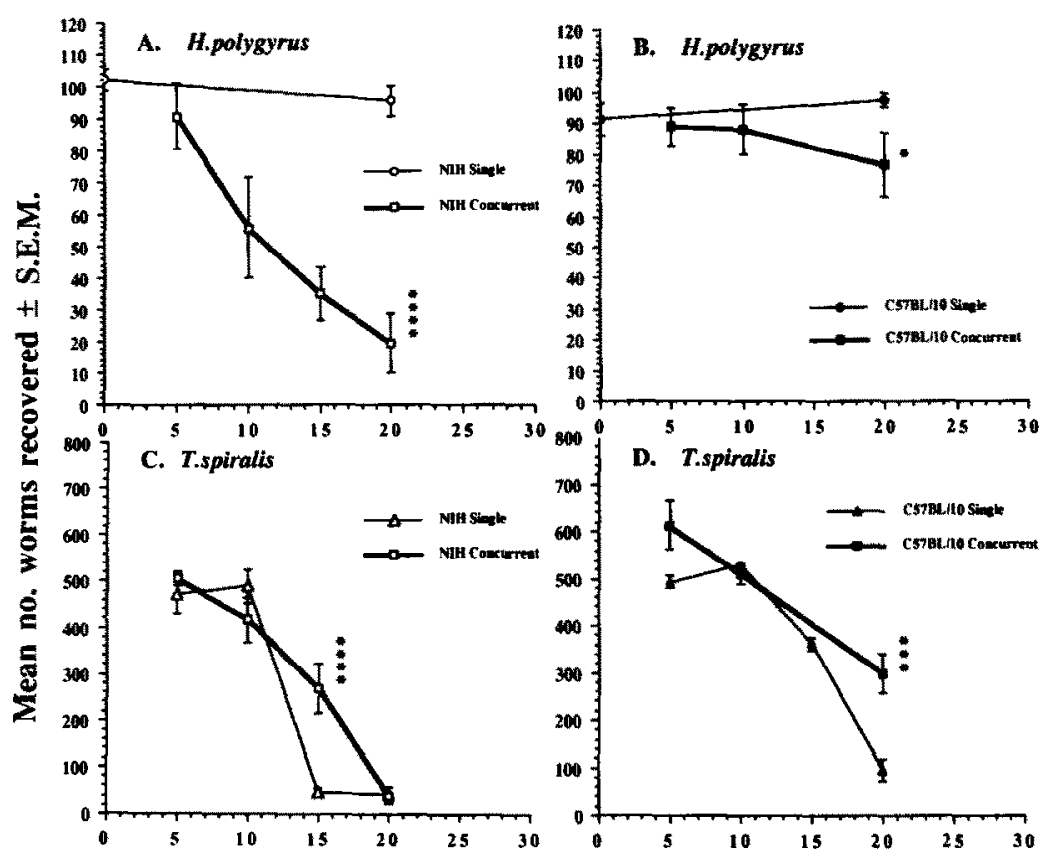

\section{Days after infection with Trichinella spiralis}

Fia. 6. Comparison of the effect on $H$. polygyrus of primary responses to $T$. spiralis in fast and slow responder mouse strains (Expt 17). A,B. Groups of male NIH or C57BL/10 mice were infected with 100 L3 of H. polygyrus on day - 14, challenged with 600 muscle larvae of $T$. spiralis on day 0 and were killed on the days shown $(n=6-7)$ for worm counts. C,D. Additional control groups from both strains monitored the course of primary infection with $T$, spiralis in the presence $(n=6-7$ on each occasion) and absence ( $n=3$ on each occasion) of $H$. polygyrus. Statistical analysis of results: Comparisons of $M W R$ s between groups within strains killed on the same day were made using the Mann-Whitney $U$ test and for the groups marked: $P=0.05$;

$$
* * 0.02 P \geqslant 0.01 ; * * * 0.01>P \geqslant 0.001 \text {. }
$$

$H$. polygyrus (Behnke et al., 1978) and therefore, $H$. polygyrus infections were kept low (100-150 larvae in most experiments, although some utilized higher doses, e.g. Expt 1). Only some of the data are shown. Thus Fig. 1 shows that during Expt 1, there was no major effect on the expulsion of $T$. spiralis, although a few larvae did persist longer in the concurrent infection group. However, Figs. 5 and 6 show quite clearly that $T$. spiralis did persist longer in concurrently infected animals despite the low doses of $H$. polygyrus which were used on those occasions. In most of the remaining experiments for which we do not present quantitative data, $T$. spiralis and $T$. pseudospiralis persisted longer in the concurrent infection groups compared with mice infected only with Trichinella spp.

\section{DISCUSSION}

Despite the inability of most mouse strains to expel primary worm burdens of $H$. polygyrus as rapidly as $T$. spiralis, the experiments reported in this paper clearly establish that two such strains (NIH and C57BL/10) have the capacity to mount an intestinal inflammatory response of sufficient intensity to cause the expulsion of many, in some experiments the majority, of $H$. polygyrus. Thus the important conclusion from our experiments is that these mouse strains have the components necessary to mount an intestinal inflammatory response capable of driving the adult worms out but normally fail to express the response and in consequence tolerate chronic primary infections with $H$. polygyrus. This conclusion again focuses attention on the importance of the immunomodulatory strategy utilized by adult $\boldsymbol{H}$. polygyrus in avoiding host immunity and it emphasizes that this strategy is essential to the parasite because $H$. polygyrus is susceptible to the non-specifically acting effectors of inflammatory responses which it has failed to prevent.

Our experiments defined the conditions under which expulsion of $H$. polygyrus could occur. The intensity of the concurrent $T$. spiralis infection was an important factor, because although some loss occurred when a low intensity $T$. spiralis infection was used, this was not always predictable and consistent effects 


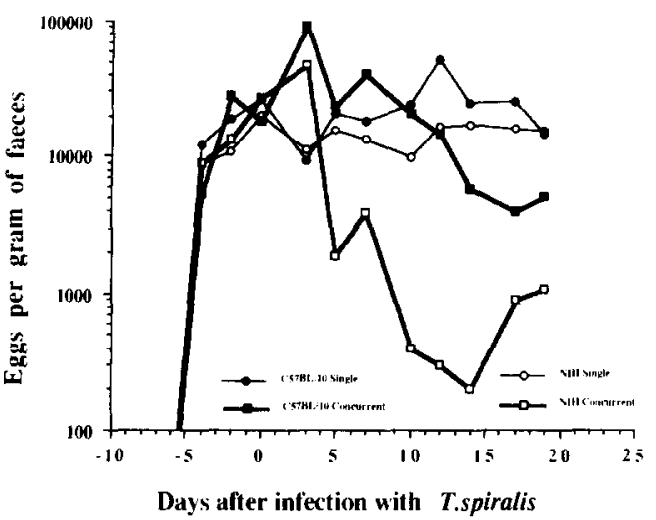

FIG. 7. Faccal egg counts (H. polygyrus) during concurrent primary infections with $T$. spiralis in fast and slow responder mouse strains (Expt 17). For details of this experiment and explanation of the key denoting groups see legend to Fig. 6.

were observed only when the higher intensity infections were employed. There was a significant negative correlation between the proportion of $H$. polygyrus surviving the inflammatory phase of the $T$. spiralis challenge and the dose of $T$. spiralis administered, although this relationship was not linear as seen in Fig. 2. We explored also the effect of T.pseudospiralis which is reputed to be less pathogenic than $T$. spiralis (Przyjalkowski et al., 1981; Przyjalkowski \& Pykalo, 1988 ) and to persist for longer after primary infection. However, NIH mice expel both species at about the same time and our experiments suggest that the inflammatory effect generated by both Trichinella spp. is comparably detrimental to the survival of $H$. polygyrus in concurrently infected animals.

One of the interesting observations was the variation in the proportion of $H$. polygyrus climinated when the relative timing of infection with the two species was varied. The proportion of $H$. polygyrus expelled declined as larvae were given nearer to the time of infection with $T$. spiralis. This reduction can be explained by the position of its larval stages. When mice were infected with $H$. polygyrus on days 0-5, L3 would have penetrated deeply into their normal site of development in the muscularis mucosa, and would have been relatively secure from expulsion at the time of peak inflammation, i.e. days 5-10, emerging from this sitc only after their normal development had been completed, some 8-10 days later (Bryant, 1973; Sukhdeo, O'Grady \& Hsu, 1984). By this time conditions in the gut lumen would have returned almost to normal. The subsequent decline in survival when $H$. polygyrus larvae were given after $T$. spiralis on days 510 is explained by a failure to establish, since in these animals $H$. polygyrus L3 larvae would have been attempting to exsheath and penetrate the intestinal mucosa at the peak of the inflammatory phase. Finally the second decline in the proportion of $H$. polygyrus being eliminated, occurring when larvae were given on days $11-15$, is explained by the less inflamed environment following the expulsion of $T$. spiralis.

The final two series of experiments exploited situations in which the inflammatory response to $T$. spiralis was known to be altered in time. Thus mice experiencing a secondary $T$. spiralis infection mount a vigorous rapid inflammatory response (Wakelin \& Lloyd, 1976; Alizadeh \& Wakelin, 1982). In contrast poor responder mice such as $\mathrm{C} 57 \mathrm{BL} / 10$ reject $T$. spiralis more slowly, later and with less vigour than fast responders such as NIH (Wakelin, 1980). The observations that adult $H$. polygyrus were lost earlier in NIH mice experiencing a secondary response to $T$. spiralis and considerably later in $\mathrm{C} 57 \mathrm{BL} / 10$ mice are in accordance with our hypothesis that it is the non-specific effectors of the inflammatory response which make life untenable for $H$. polygyrus.

One more approach could have been used to confirm the requirement for an active immune system and the expression of an inflammatory response but this was not available to us. Immunodepression of concurrently infected mice could have been exploited to demonstrate that in the absence of an inflammatory response both species can co-exist together. However, the combination of immunodepression and two relatively pathogenic intestinal parasites is more than mice can tolerate at the doses that are required. Small-scale exploratory pilot experiments convinced us that this approach was not one which we wished to pursue further.

The reciprocal interaction, i.e. the effect of $H$. polygyrus on the expulsion of $T$. spiralis, was not an objective of this work but nevcrtheless our data confirm that there is an immunodepressive influence of $H$. polygyrus which was detectable in some experiments despite out attempts to minimize the reciprocal interaction. The majority of experiments were carried out with low doses of $H$. polygyrus, large enough for a reduction in the worm burden to be detected but low enough not to cause a major impairment of the response to $T$. spiralis. Behnke et al. (1978) demonstrated that expulsion of $T$. spiralis was most consistently impaired when the infection intensity of H. polygyrus cxceeded 200 adult worms and in most of these earlier experiments doses of 200-400 worms were used. However, in spite of our precautions it was quite clear from some of the results we have presented here, as well as from the large amount of additional data arising from necessary control groups which we have not included, that the immunodepressive influence of $H$. polygyrus was still evident in concurrently infected 
mice and that there was a degree of impairment of the response to $T$. spiralis. Nevertheless, $T$. spiralis were rejected and the response carried with it a proportion of $H$. polygyrus. However, it was equally interesting to observe that in most experiments some $H$. polygyrus managed to survive. Even when the infection intensity of $T$. spiralis was high and over $90 \%$ of $H$. polygyrus were lost, the remainder survived and continued to produce eggs as normal. Clearly, the intestinal effectors were not $100 \%$ effective against $H$. polygyrus even at the best of times demonstrating again the resilience and adaptability of this parasite in the face of inflammatory host protective responses.

In summary, our experiments have demonstrated unequivocally that mice can reject $H$. polygyrus through the same combination of intestinal inflammatory effectors which are normally invoked in response to $T$. spiralis. Most worms are susceptible to these effectors and when located in an inflamed intestine cannot escape from their damaging influence. However, a variable proportion of worms always survived even in immune mice responding to homologous challenge with $T$. spiralis in which intestinal inflammation was at its most intense. Chronic primary infections of $H$. polygyrus most likely maintain themselves by preventing host inflammatory effectors from being triggered in the first place and it is conceivable that in some mice aggregations of adult worms exerted sufficient local immunomodulatory influence to minimize the release of inflammatory mediators in the vicinity of the site occupied by the parasites (Behnke, 1978). Alternatively a subpopulation of worms may have been more resistant than the remainder even when immune effectors were triggered. This resilience in the face of host effectors could be attributable to the worm's relatively high free oxygen radical scavenging enzymes (Smith \& Bryant, 1986) or to other, as yet undetermined qualities but either way it is likely that the parasite has a second line of defence against host immune responses.

Acknowledgements-We would like to thank Prof. P. N. R. Usherwood for the provision of facilities for this research project in the Department of Zoology of Nottinghan University. WC was supported by a short study leave grant from the British Council. The work was financed by MRC grants G8100159T \& G8328675T to JMB. We are indebted to Mr K. Cosgrove for supervision over the husbandry of our experimental animals.

\section{REFERENCES}

Alizadeh H. \& Wakelin D. 1982. Comparison of rapid expulsion of Trichinella spiralis in mice and rats. International Journal for Parasitology 12: 65-73.

Beaver P. C. 1988. Light, long-lasting Necator infection in a volunteer. American Journal of Tropical Medicine and
Hygiene 39: 369-372.

BehnKe J, M., Bland P. W. \& WaKelin D. 1977. The effect of the expulsion phase of Trichinella spiralis on Hymenolepis diminuta infection in mice. Parasitology 75: 79-88.

BehnKe J. M., WaKelin D. \& WiLson M. M. 1978. Trichinella spiralis: delayed rejection in mice concurrently infected with Nematospiroides dubius. Experimental Parasitology 46: $121-130$.

Behnke J. M. \& Parish H. A. 1979. Nematospiroides dubius: arrested development of larvae in immune mice. Experimental Parasitology 47: 116-127.

Behnke J. M. 1987. Evasion of immunity by nematode parasites causing chronic infections. Advances in Parasitology 26: 1-71.

BRYANT V. 1973. The life cycle of Nematospiroides dubius, Baylis, 1926 (Nematoda: Heligmosomidae). Journal of Helminthology 48: 263-268.

CABAJ W. 1989. Intestinal and muscle phases of Trichinella pseudospiralis infection in $\mathrm{C} 3 \mathrm{H}$ mice previously infected with Nematospiroides dubius. Acta Parasitologica Polonica 34: 181-189.

Christensen N. O., Nansen P., Fagbemi B. O. \& Monrad J. 1987. Heterologous antagonistic interactions between helminths and between helminths and protozoans in concurrent experimental infection of mammalian hosts. Parasitology Research 73: 387-410.

Christie P. R., Wakelin D. \& Wilson M. M. 1979. The effect of the expulsion phase of Trichinella spiralis on Hymenolepis diminuta infection in rats. Parasitology 78: 323-330.

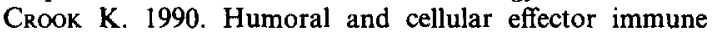
responses against parasites. In: Parasites: Immunity and Pathology. The Consequences of Parasitic Infection in Mammals (Edited by BeHNKe J. M.), pp. 89-119. Taylor and Francis, London.

Dehlawi M. S., Wakelin D. \& Behnke J. M. 1987. Suppression of mucosal mastocytosis by infection with the intestinal nematode Nematospiroides dubius. Parasite Immunology 9: 187-194.

Douch P. G. F., Harrison G. B. L., Buchanan L. L. \& Greer K. S. 1983. In vitro bioassay of sheep gastrointestinal mucus for nematode paralysing activity mediated by a substance with some properties characteristic of SRS-A. International Journal for Parasitology 13: 207-212.

Durette-Desset M. C., Kinsella J. M. \& Forrester D. J. 1972. Arguments en faveur de la double origine des nématodes nearctiques du genre Heligmosomoides Hall, 1916. Annales de Parasitologie humaine et comparée 47: 365-382.

Ferretti G., Gabriele F., Palmas C. \& Wakelin D. 1984. Interactions between Trichinella spiralis and Hymenolepis nana in the intestine of the mouse. International Journal for Parasitology 14: 29-33.

Grencis R. K., Riedlinger J. \& Wakelin D. 1985. L3T4positive $\mathrm{T}$ lymphoblasts are responsible for transfer of immunity to Trichinella spiralis in mice. Immunology 56: 213-218.

Howard R. J., Christie D., Wakelin D., Wilson M. M. \& BEHNKE J. M. 1978. The effect of concurrent infection with Trichinella spiralis on Hymenolepis microstoma in mice. Parasitology 77: 273-279. 
JENKINS S. N. \& BeHNKe J. M. 1977. Impairment of primary expulsion of Trichuris muris in mice concurrently infected with Nematospiroides dubius. Parasitology 75: 71-78.

KENNEDY M. W. 1980. Immunologically mediated, nonspecific interactions between the intestinal phases of Trichinella spiralis and Nippostrongylus brasiliensis in the mouse. Parasitology 80: 61-72.

McKean P. G. \& Pritchard D. I. 1989. The action of a mast cell protease on the cuticular collagens of Necator americanus. Parasite Immunology 11: 293-297.

MoQbel R. \& MacDonald A. J. 1990. Immunological and inflammatory responses in the small intestine associated with helminthic infections. In: Parasites: Immunity and Pathology. The Consequences of Parasitic Infection in Mammals (Edited by BeHNKE J. M.), pp. 249-282. Taylor and Francis, London.

Ovington K. S. 1987. Nippostrongylus brasiliensis: physiological and metabolic responses of rats to primary infection. Experimental Parasitology 63: 10-20.

Palmer E. D. 1955. Course of egg output over a 15 year period in a case of experimentally induced necatoriasis americanus, in the absence of hyperinfection. American Journal of Tropical Medicine and Hygiene 4: 756-757.

Przyjalkowski Z., Starzynski S., Pykalo R. \& Cabaj W. 1981. Serological and pathological changes in germfree and conventional mice in single or mixed Trichinella spiralis and $T$. pseudospiralis infections. In: Recent Advances in Germfree Research (Edited by SASAKI S.), pp. 447-452. Tokai University Press, Japan.

Przyjalkowski Z. \& Pykalo R. 1988. Histopathological changes in the course of single and mixed Trichinella spiralis and $T$. pseudospiralis infections in germfree and conventional mice. Acta Parasitologica Polonica 33: 5969.

Robinson M., Wahid F., Behnke J. M. \& Gilbert F. S. 1989. Immunological relationships during primary infection with Heligmosomoides polygyrus (Nematospiroides dubius): dose-dependent expulsion of adult worms. Parasitology 98: 115-124.

Rothwell T. L. W. 1989. Immune expulsion of parasitic nematodes from the alimentary tract. International Journal for Parasitology 19: 139-168.

SHAw J. L. \& Moss R. 1989. The role of parasite fecundity and longevity in the success of Trichostrongylus tenuis in low density red grouse populations. Parasitology 99: 253258.

SMith N. C. \& Bryant C. 1986. The role of host generated free radicals in helminth infections: Nippostrongylus brasiliensis and Nematospiroides dubius compared. International Journal for Parasitology 16: 617-622.

SMITH N.C. \& BRYant C. 1989a. Free radical generation during primary infections with Nippostrongylus brasiliensis. Parasite Immunology 11: 147-160.

SMith N. C. \& Bryant C. 1989 b. The effect of antioxidants on the rejection of Nippostrongylus brasiliensis. Parasite Immunology 11: 161-167.

Sokal R. R. \& Rohlf F. J. 1969. Biometry. Freeman, San Francisco.

Stewart G. L., Wood B. G. \& Boley R. B. 1985. Modulation of host response by Trichinella spiralis. Parasite Immun$\operatorname{olog} y$ 7: 223-233.

Stewart G. L., Mann M. A., Ubelaker J. E., McCarthy J. L. \& WooD B. G. 1988. A role for elevated plasma corticosterone in modulation of host response during infection with Trichinella pseudospiralis. Parasite Immunology 10: 139-150.

Sukhdeo M. V. K., O'Grady R. T. \& Hsu S. C. 1984. The site selected by the larvae of Heligmosomoides polygyrus. Journal of Helminthology 58: 19-23.

WAKELIN D. \& LLOYD M. 1976. Immunity to primary and challenge infections of Trichinella spiralis in mice: a reexamination of conventional parameters. Parasitology 72 : 173-183.

WAKELIN D. 1978. Genetic control of susceptibility and resistance to parasitic infections. Advances in Parasitology 16: $219-308$.

WAKELIN D. 1980. Genetic control of immunity to parasites. Infection with Trichinella spiralis in inbred and congenic mice showing rapid and slow responses to infection. Parasite Immunology 2: 85-90. 